KYUSHU-HET 57

OUHEP-01-1

\title{
Search for T-violation in Neutrino Oscillation with the Use of Muon Polarization at a Neutrino Factory
}

\author{
Toshihiko Ota* \\ Department of Physics, Kyushu University, \\ Hakozaki, Higashi-ku, Fukuoka 812-8581, Japan \\ Joe Sato ${ }^{\dagger}$ \\ Research Center for Higher Education, Kyushu University, \\ Ropponmatsu, Chuo-ku, Fukuoka 810-8560, Japan \\ and \\ Yoshitaka Kuno \\ Department of Physics, Osaka University, \\ Machikane-yama 1-1, Toyonaka, Osaka 560-0043, Japan
}

\begin{abstract}
A possibility to search for T-violation in neutrino oscillation with the use of muon polarization is studied. The sensitivity to T-violation is examined with various magnitudes of muon polarization as a function of muon energy and long-baseline distances.
\end{abstract}

\section{Introduction}

In this note, we discuss a possibility to discover the CP-violating effect in the leptonic sector [1] at a neutrino factory. Observation of CP-violation would imply the measurement of an imaginary part of the couplings in the Lagrangian associated with leptons [2]. There are two ways to study $\mathrm{CP}$ violation. They are

1. to study a difference between the CP conjugate modes, or

2. to study a difference between the $\mathrm{T}$ conjugate modes.

*e-mail address: toshi@higgs.phys.kyushu-u.ac.jp

$\dagger$ e-mail address: joe@rc.kyushu-u.ac.jp

${ }^{\ddagger}$ e-mail address: kuno@phys.sci.osaka-u.ac.jp 
The first method is to observe the difference between the particle and its anti-particle. In a neutrino factory, it can be studied by comparing the difference of the appearance event rates between $\nu_{e} \rightarrow \nu_{\mu}$ and $\bar{\nu}_{e} \rightarrow \bar{\nu}_{\mu}$ [3, 4, 9, 6, 6, 8, 9]. However, it has been pointed out that the matter effect would introduce a sizeable fake CP-odd effect [10, 11]. It is necessary to discriminate the genuine $\mathrm{CP}$ violation effect from the fake matter effect.

The second method is to observe the difference between the transitions $\nu_{e} \rightarrow \nu_{\mu}$ and $\nu_{\mu} \rightarrow \nu_{e}$. By the CPT theorem, in vacuum, observation of T-violation is identical to observation of CP-violation. Even in a terrestrial oscillation experiment, since a possible T-odd effect from asymmetric matter density profile in the earth is very small [12], this T-reversed difference reveals as a very clean signal [2, 13] on the imaginary phase in the lepton sector [2, 9, 10, 12, 13, 14, 15, 16, 17, 18, 19, 20, 21, 22, 23, 24, 25, 26.

Therefore, the comparison of T-reversed oscillation modes would give clear signature than those of CP-reversed oscillation modes. However, although it is easy to observe $\nu_{e} \rightarrow \nu_{\mu}$ oscillation, it is known to be difficult to observe $\nu_{\mu} \rightarrow \nu_{e}$ oscillation at a neutrino factory. The reason is as follows. At a neutrino factory, $\nu_{\mu}$ is generated by the decay of muons as well as $\bar{\nu}_{e}$. The former after the oscillation from $\nu_{\mu} \rightarrow \nu_{e}$, produces $e^{-} \mathrm{s}$ and the latter from muon decays produces $e^{+} \mathrm{s}$ at a detector. It is difficult to distinguish $e^{+}$from $e^{-}$after the creation of electromagnetic shower in most of the detectors being considered at present. Therefore, it is difficult to identify the oscillation events from the non-oscillation events which is larger in statistics.

It is critical to examine whether there are any solutions to solve this problem for the search for T-violation modes useful. There was one idea to identify the oscillation events by the use of polarized muons in the muon storage ring [17]. The principle of the idea is the following. Supposed that the muons in the muon storage ring have their spin polarization $P_{\mu^{-}}=-1$, there would be no $\bar{\nu}_{e}$ and only $\nu_{\mu}$ at the very forward direction along the muon momentum direction. Observation of $e$-like events at a detector would directly imply the appearance of $\nu_{\mu} \rightarrow \nu_{e}$. In reality, the muon spin polarization would not be $100 \%$. But, if the spin polarization of the muons in the muon storage ring can be changed, accordingly the neutrino energy spectrum shapes can be changed. The changes of the spectrum of neutrino and anti-neutrino are different. Therefore, in principle we could discriminate the events associated with neutrinos from those from anti-neutrino. In turn, the oscillating events and non-oscillating events can be separated.

\section{Principle}

In this section, the principle to discriminate the oscillating events by using the muon polarization is presented. The neutrino flux in the very forward direction of a $\mu^{-}$beam with its muon spin polarization $P_{\mu}$ is given by

$$
\begin{aligned}
\Phi_{\bar{\nu}_{e}} & \propto y^{2}(1-y) \cdot\left(1+P_{\mu}\right) \\
\Phi_{\nu_{\mu}} & \propto y^{2}(3-2 y)+P_{\mu} \cdot y^{2}(1-2 y)
\end{aligned}
$$


where $y \equiv E_{\nu} / E_{\mu} . E_{\mu}$ is the $\mu^{-}$energy and $E_{\nu}$ is the neutrino energy. For $\mu^{+}$, eqs.(1) and (2) can be given with changing the sign of $P_{\mu}$. From using eqs.(1) and (2), the non-oscillating event rate of $\bar{\nu}_{e} \rightarrow \bar{\nu}_{e}\left(N_{\bar{\nu}_{e} \rightarrow \bar{\nu}_{e}}\right)$ and the appearance event rate of $\nu_{\mu} \rightarrow \nu_{e}$ $\left(N_{\nu_{\mu} \rightarrow \nu_{e}}\right)$ are given by

$$
\begin{aligned}
& N_{\bar{\nu}_{e} \rightarrow \bar{\nu}_{e}}=\left(1+P_{\mu}\right) N_{3}, \\
& N_{\nu_{\mu} \rightarrow \nu_{e}}=N_{1}+P_{\mu} N_{2},
\end{aligned}
$$

respectively. Here $N_{1}, N_{2}$ and $N_{3}$ are the event numbers corresponding to each term of neutrino flux. They are typically represented by

$$
\begin{aligned}
& N_{1}=C \frac{E_{\mu}^{2}}{L^{2}} \int_{E_{\min } / E_{\mu}}^{E_{\max } / E_{\mu}} d y 2 y^{2}(3-2 y) P_{\nu_{\mu} \rightarrow \nu_{e}}\left(E_{\nu}\right) \sigma\left(E_{\nu}\right) \\
& N_{2}=C \frac{E_{\mu}^{2}}{L^{2}} \int_{E_{\min } / E_{\mu}}^{E_{\max } / E_{\mu}} d y 2 y^{2}(1-2 y) P_{\nu_{\mu} \rightarrow \nu_{e}}\left(E_{\nu}\right) \sigma\left(E_{\nu}\right) \\
& N_{3}=C \frac{E_{\mu}^{2}}{L^{2}} \int_{E_{\min } / E_{\mu}}^{E_{\max } / E_{\mu}} d y 12 y^{2}(1-y) P_{\bar{\nu}_{e} \rightarrow \bar{\nu}_{e}}\left(E_{\nu}\right) \bar{\sigma}\left(E_{\nu}\right),
\end{aligned}
$$

where $P_{\nu_{\mu} \rightarrow \nu_{e}}, P_{\bar{\nu}_{e} \rightarrow \bar{\nu}_{e}}$ are oscillation probabilities, $\sigma(\bar{\sigma})$ is the detection rate including the neutrino (anti-neutrino) cross section and signal efficiency, $E_{\min }\left(E_{\max }\right)$ is minimum (maximum) neutrino energy observed in this assumption, $L$ is the baseline length, and $C$ is the constant proportionate to the decay muon number and the detector mass. From these equations, the rate of $e$-like events, which is the sum of $N_{\bar{\nu}_{e} \rightarrow \bar{\nu}_{e}}$ and $N_{\nu_{\mu} \rightarrow \nu_{e}}$, can be given by

$$
\begin{aligned}
N_{e-\text { like }} & =\beta_{0}+P_{\mu} \beta_{1}, \\
\beta_{0} & \equiv N_{1}+N_{3}, \\
\beta_{1} & \equiv N_{2}+N_{3} .
\end{aligned}
$$

Note that for $P_{\mu}=-1, N_{e-\text { like }}$ becomes equal to $N_{\nu_{\mu} \rightarrow \nu_{e}}$ itself from eq.(3). The estimate of the $e$-like event rate at $P_{\mu}=-1$ is essentially the measurement of $N_{\nu_{\mu} \rightarrow \nu_{e}}$. In another words, if $\beta_{0}$ and $\beta_{1}$ can be estimated, the direct observation of $N_{\nu_{\mu} \rightarrow \nu_{e}}$ could be made in principle. This is shown in Fig.11.

\section{Procedure}

In this section, the procedure to estimate the appearance event rate, $N_{\nu_{\mu} \rightarrow \nu_{e}}$, at $P_{\mu}=-1$ from the measurements is given. In reality, the muons in the muon storage ring have some distribution of their polarization. It is assumed that a number of the muons of their muon polarization of $P_{\mu_{i}},(i=1 \cdots n)$ is $N_{i}\left(\equiv N_{\mu} / f_{i}\right),(i=1 \cdots n)$. They yield $y_{i} e$-like 


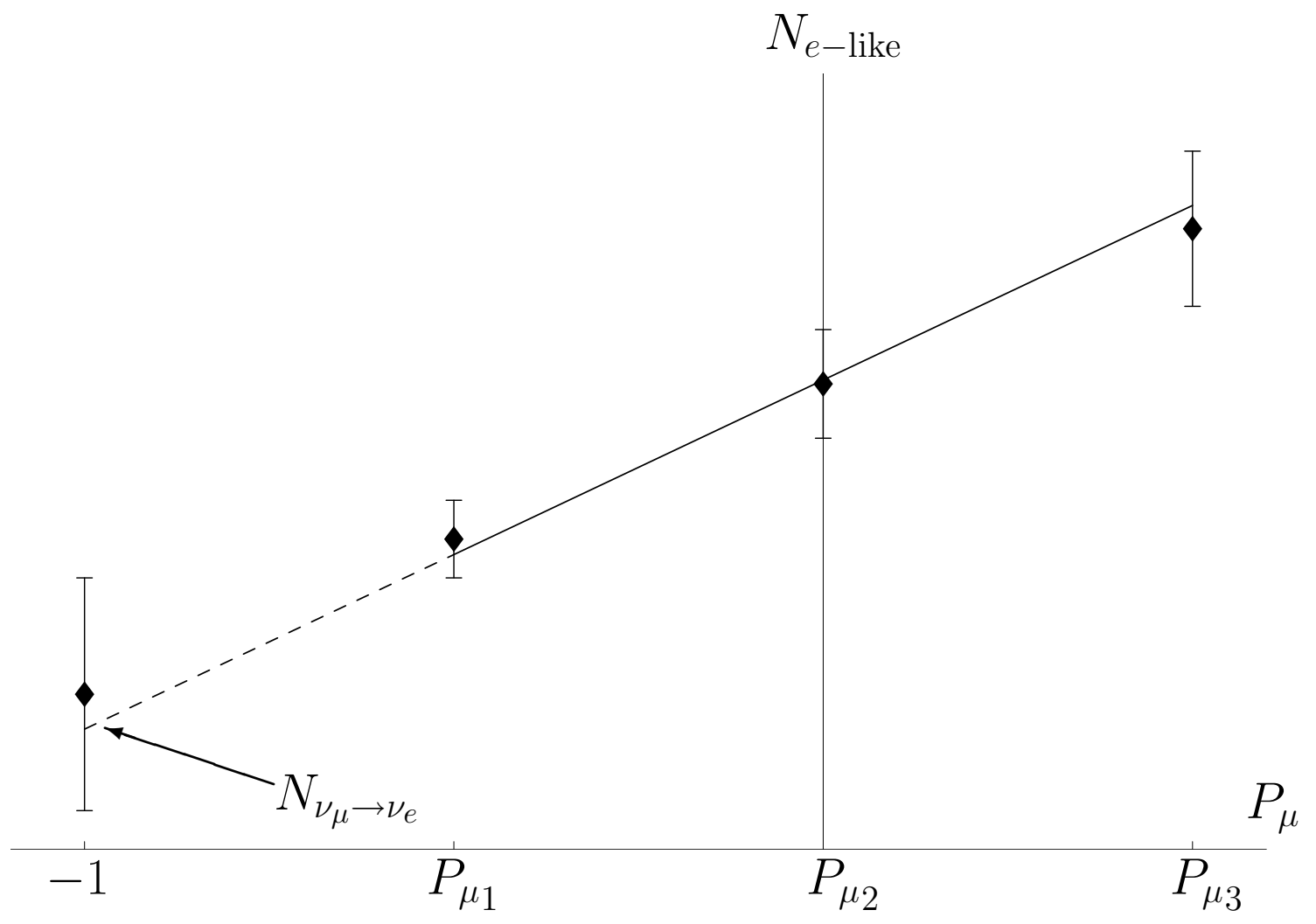

Figure 1: Graphical view of the idea. We have observations of $e$-like event with partially polarized muon beams and from them we estimate the event rate at $P_{\mu}=-1$.

events. $N_{\mu}$ is a total number of the muons in the ring. For those, eq.(8) can be rewritten by

$$
\begin{aligned}
N_{e-\text { like }} & =\beta_{0}^{*}+P_{\mu}^{*} \beta_{1}, \\
\beta_{0}^{*} & \equiv \beta_{0}+\bar{P}_{\mu} \beta_{1}, \\
P_{\mu}{ }^{*} & \equiv P_{\mu}-\bar{P}_{\mu}=-1-\bar{P}_{\mu}, \\
\bar{P}_{\mu} & \equiv \frac{1}{F} \sum_{i} \frac{P_{\mu_{i}}}{f_{i}}, \\
F & \equiv \sum_{i} \frac{1}{f_{i}} .
\end{aligned}
$$

The expectation values of $y_{i}, \bar{y}_{i}$ is given by

$$
f_{i} \bar{y}_{i}=\beta_{0}+P_{\mu_{i}}^{*} \beta_{1}
$$

It is assumed that $y_{i}$ 's are large enough so that it would follow the normal distribution

\footnotetext{
${ }^{1}$ In the following, we denote $\beta_{0}^{*}$ by $\beta_{0}$.
} 
of $\mathrm{N}\left(\bar{y}_{i}, \bar{y}_{i}\right)$. In this case, the likelihood function to estimate $\beta_{0}$ and $\beta_{1}$ is given by

$$
-\log L=\sum_{i} \frac{\left\{f_{i} y_{i}-\left(\beta_{0}+P_{\mu_{i}^{*}}^{*} \beta_{1}\right)\right\}^{2}}{2 f_{i}^{2} y_{i}} .
$$

From this likelihood function, the equations for the estimates of $\beta_{0}$ and $\beta_{1}$ can be give by

$$
\begin{aligned}
0=-\frac{\partial \log L}{\partial \beta_{0}} & =\beta_{0} \sum_{i} \frac{1}{f_{i}^{2} y_{i}}+\beta_{1} \sum_{i} \frac{P_{\mu_{i}}^{*}}{f_{i}^{2} y_{i}}-\sum_{i} \frac{1}{f_{i}} \\
0=-\frac{\partial \log L}{\partial \beta_{1}} & =\beta_{0} \sum_{i} \frac{P_{\mu_{i}}^{*}}{f_{i}^{2} y_{i}}+\beta_{1} \sum_{i} \frac{\left(P_{\mu_{i}}^{*}\right)^{2}}{f_{i}^{2} y_{i}}-\sum_{i} \frac{P_{\mu_{i}}^{*}}{f_{i}} \\
& =\beta_{0} \sum_{i} \frac{P_{\mu_{i}}^{*}}{f_{i}^{2} y_{i}}+\beta_{1} \sum_{i} \frac{\left(P_{\mu_{i}}^{*}\right)^{2}}{f_{i}^{2} y_{i}} .
\end{aligned}
$$

Here the definitions of $P_{\mu}{ }^{*}$ by eq.(13), and of $\bar{P}_{\mu}$ by eq.(14) are used. Also the last term in the first line of eq.(19) is removed. Using these eqs.(18) and (19), $\beta_{0}$ and $\beta_{1}$ in terms of $y_{i}$ can be estimated as follows:

$$
\begin{aligned}
& \beta_{0}=\frac{F}{D} \sum_{i} \frac{\left(P_{\mu_{i}}^{*}\right)^{2}}{f_{i}^{2} y_{i}} \text { and } \\
& \beta_{1}=-\frac{F}{D} \sum_{i} \frac{P_{\mu_{i}}^{*}}{f_{i}^{2} y_{i}},
\end{aligned}
$$

where

$$
D \equiv\left(\sum_{i} \frac{1}{f_{i}^{2} y_{i}}\right)\left(\sum_{i} \frac{\left(P_{\mu_{i}}^{*}\right)^{2}}{f_{i}^{2} y_{i}}\right)-\left(\sum_{i} \frac{P_{\mu_{i}}^{*}}{f_{i}^{2} y_{i}}\right)^{2}
$$

Thus, $N_{\nu_{\mu} \rightarrow \nu_{e}}$ at $P_{\mu}=-1$ can be estimated using eqs.(20) and (21) as

$$
N_{\nu_{\mu} \rightarrow \nu_{e}}=\beta_{0}+\left(-1-\bar{P}_{\mu}\right) \beta_{1} .
$$

The statistical error on this estimated value is now evaluated. Formally, the variance of the estimate is given by

\footnotetext{
${ }^{2}$ Exactly speaking, the denominator of eq. (17) $f_{i}^{2} \bar{y}_{i}$. However, for simplicity, under the assumption that $y_{i}$ 's are large, it can be replaced with $f_{i}^{2} y_{i}$. This is justified since the non-oscillating event rates $N_{\bar{\nu}_{e} \rightarrow \bar{\nu}_{e}}$ at any polarization are large. (It does not depend on whether there are enough appearance event rate $N_{\nu_{\mu} \rightarrow \nu_{e}}$ as long as the polarization is not very close to -1 .)

${ }^{3}$ The likelihood function with Poisson distribution can be also treated. By this, the same function as eq.(17) can be obtained under the assumption that $y_{i}$ 's are large enough.
} 
$V\left(N_{\nu_{\mu} \rightarrow \nu_{e}}\right)=V\left(\beta_{0}+\left(-1-\bar{P}_{\mu}\right) \beta_{1}\right)=V\left(\beta_{0}\right)+\left(-1-\bar{P}_{\mu}\right)^{2} V\left(\beta_{1}\right)+2\left(-1-\bar{P}_{\mu}\right) V\left(\beta_{0}, \beta_{1}\right),(24$

where $V(\beta)$ is the variance of $\beta$, and $V\left(\beta_{0}, \beta_{1}\right)$ is the covariance of $\beta_{0}, \beta_{1}$.

It is impossible to evaluate the variance of $N_{\nu_{\mu} \rightarrow \nu_{e}}$ using eqs.(20) and (21) directly. Therefore, it is approximated by the inverse matrix of the expectation value of Fisher's information.f Fisher's information matrix is given by

$$
-\frac{\partial^{2}}{\partial \beta_{i} \partial \beta_{j}} \log L=\left(\begin{array}{cc}
\sum_{i} \frac{1}{f_{i}^{2} y_{i}} & \sum_{i} \frac{P_{\mu_{i}}^{*}}{f_{i}^{2} y_{i}} \\
\sum_{i} \frac{P_{\mu_{i}}^{*}}{f_{i}^{2} y_{i}} & \sum_{i} \frac{\left(P_{\mu_{i}}^{*}\right)^{2}}{f_{i}^{2} y_{i}}
\end{array}\right),
$$

and hence the variance matrix is given by

$$
\frac{1}{D}\left(\begin{array}{cc}
\sum_{i} \frac{\left(P_{\mu_{i}}^{*}\right)^{2}}{f_{i}^{2} y_{i}} & -\sum_{i} \frac{P_{\mu_{i}}^{*}}{f_{i}^{2} y_{i}} \\
-\sum_{i} \frac{P_{\mu_{i}}^{*}}{f_{i}^{2} y_{i}} & \sum_{i} \frac{1}{f_{i}^{2} y_{i}}
\end{array}\right)
$$

Thus, the estimate of $N_{\nu_{\mu} \rightarrow \nu_{e}}$ has its variance of

$$
\begin{aligned}
V\left(N_{\nu_{\mu} \rightarrow \nu_{e}}\right) & =\frac{1}{D}\left\{\sum_{i} \frac{\left(P_{\mu_{i}}^{*}\right)^{2}}{f_{i}^{2} y_{i}}+\left(-1-\bar{P}_{\mu}\right)^{2} \sum_{i} \frac{1}{f_{i}^{2} y_{i}}-2\left(-1-\bar{P}_{\mu}\right) \sum_{i} \frac{P_{\mu_{i}}^{*}}{f_{i}^{2} y_{i}}\right\} \\
& =\frac{1}{F}\left[2 N_{\nu_{\mu} \rightarrow \nu_{e}}+N_{\bar{P}_{\mu}}\left\{\left(-1-\bar{P}_{\mu}\right)^{2} \frac{\sum_{i} 1 / f_{i}^{2} y_{i}}{\sum_{i}\left(P_{\mu_{i}}^{*}\right)^{2} / f_{i}^{2} y_{i}}-1\right\}\right] .
\end{aligned}
$$

Here $N_{\bar{P}_{\mu}}$ is the estimate of the event rate at $P_{\mu}=\bar{P}_{\mu}$ when $N_{\mu^{-}} \mu^{-}$s are used.

\section{Numerical Analysis}

In the analytical treatment shown above, several assumptions are made. For instance, it is assumed that the likelihood function of the $\beta$ 's is given by eq.(17), and the variance of the estimate is given by the inverse of the Fisher's information matrix.

In this section, numerical calculations is given. What we have to calculate numerically is the extrapolated value at $P_{\mu}=-1$ which indeed coincides with the theoretical expectation value $\left.\bar{y}\right|_{P_{\mu}=-1}$ and the variance of it which is actually given by eq.(28).

The algorithm for this numerical analysis is as follows:

\footnotetext{
${ }^{4}$ Strictly speaking, the inverse matrix of the expectation value of Fisher's information gives the lower limit of the corresponding variance. However if the likelihood function is constructed well then it coincide with the variance and hence we use this approximation. We also approximate the expectation value of the Fisher's information by eq.(25) since it is assumed that $y_{i}$ 's are large number enough.
} 
step 0. Select several values of the polarization $P_{\mu_{i}}$ for the measurements,

step 1. Fix all the parameters, such as the theoretical parameters (e.g. mixing angle), a muon energy and so on, and then calculate the expectation of event rates in each polarization values $\left(\bar{y}_{i}\right)$.

step 2. Generate the "actual" events of $y_{i}$ according to Poisson distribution with its expectation value $\bar{y}_{i}$

step 3. Substitute these event sets into eqs.(20), (21), and find the extrapolated value at $P_{\mu}=-1$ from eq. (23) by using these $\beta$ 's.

step 4. Iterate the step 2 and step 3 in several times to find the type of distribution of the extrapolated value.

This virtual measurements for various parameters were made. It is found that the extrapolated value follows the normal distribution with its mean value $\left.\bar{y}\right|_{P_{\mu}=-1}$ and its variance given by eq. (28).

\section{Sensitivity for $\mathbf{T}$ Violation}

In this section, the sensitivity to T-violation using the present procedure is discussed to find the statistics to see the T-violation effect.

$$
\chi^{2}(n)=\sum_{i}^{n} \frac{\left(N_{0 i} \bar{N}_{i}-\bar{N}_{0 i} N_{i}\right)^{2}}{N_{0 i}^{2} V_{i}+\bar{N}_{0 i}^{2} N_{i}}
$$

where

$$
\begin{gathered}
N: N_{\nu_{e} \rightarrow \nu_{\mu}}, \\
\bar{N}: N_{\nu_{\mu} \rightarrow \nu_{e}},
\end{gathered}
$$

and the subscript 0 indicates the estimate of the event rate with $\mathrm{CP}$ violating phase $\delta=\delta_{0} \equiv\{0, \pi\}$ and subscript $i$ represents $i$ th energy bin. Note that in the denominator of $\chi^{2}$ there is $V$. Although $V$ is given by eq.(28) theoretically, it is calculated by using the numerical method in section 1 . To confirm that $\mathrm{T}$ violation can be observed at $90 \%$ confidence level, $\chi^{2}(n)>\chi_{90 \%}^{2}(n)$ is required. Suppose that three measurements can be adopted with different muon polarization values $\left(P_{\mu_{i}}=\left\{-P_{\mu}, 0, P_{\mu}\right\}\right)$, the sensitivity of $90 \%$ confidence level can be calculated. It is shown in Fig.2. We chose the most sensitive method among $n=1,3,5$ in each $\left(E_{\mu}, L\right)$ region. The usage of statistics is referred in Ref. [11] in detail.

Here, it is discussed how many neutrinos from muon decays should be needed to observe the T-violation effect. First of all, for simplicity, $N_{i}=N_{\mu^{+}}$is assumed. Here, the following theoretical parameters are used; $\sin \theta_{12}=0.5, \sin \theta_{23}=1 / \sqrt{2}, \sin \theta_{13}=0.1$, 

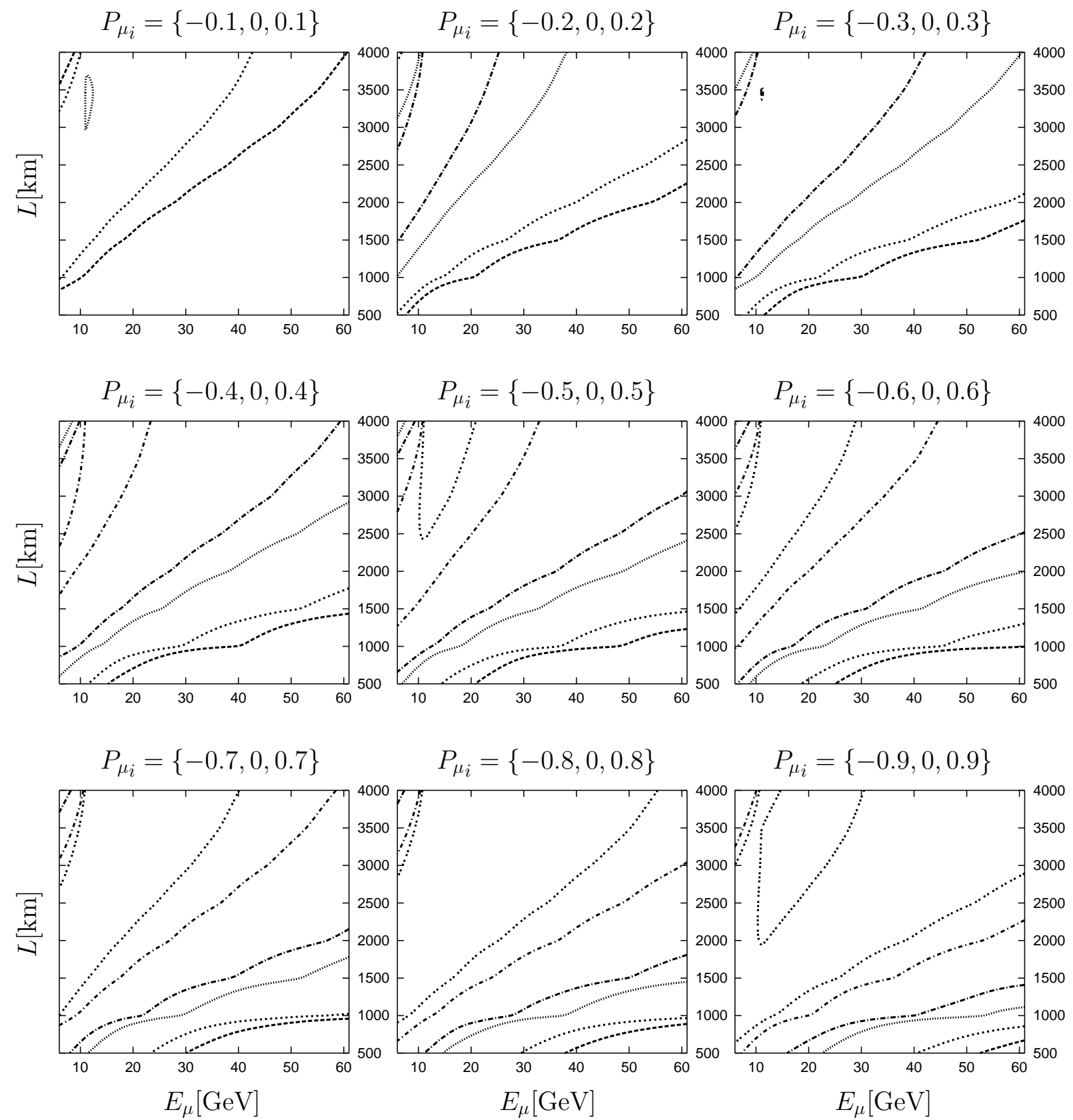

Figure 2: Required data sizes in the unit of $10^{21}$ muon decays $\times 100$-kt detector to obtain a sensitivity of $90 \%$ confidence level are plotted as a function of muon energy and baseline length for various sets of muon polarizations $P_{\mu_{i}}$. The meaning of each line is explained

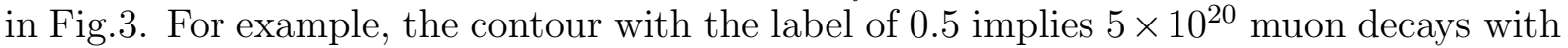
a 100-kt detector is used. For each case, three measurements with different $P_{\mu_{i}}$ values are assumed. These three values of the muon polarization are shown for each plot. If the muon polarization is high, the necessary data size is smaller. 

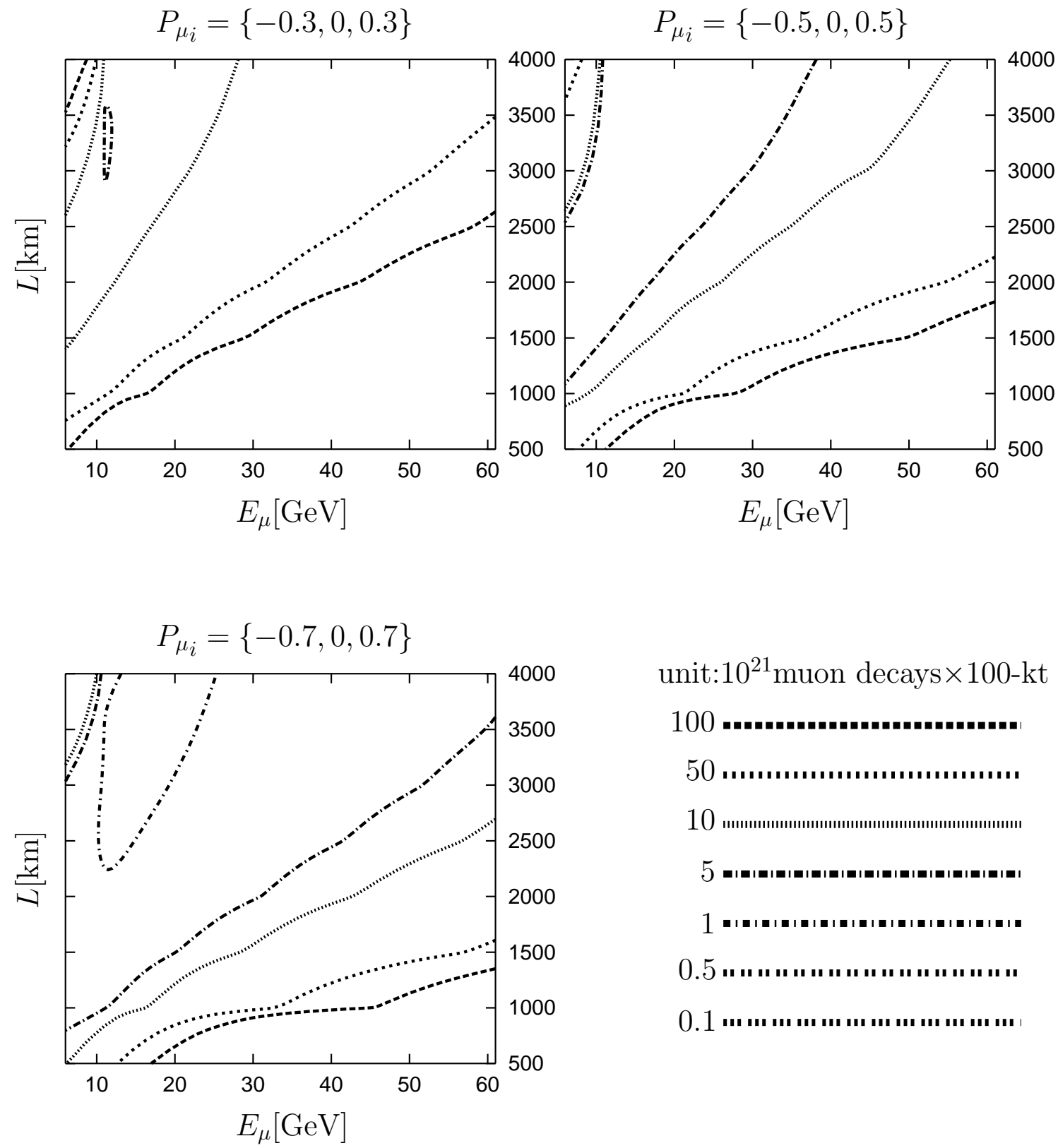

unit: $10^{21}$ muon decays $\times 100-\mathrm{kt}$

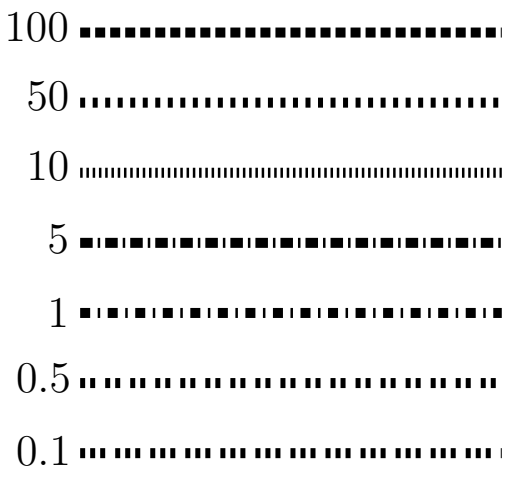

Figure 3: Same as Fig.2, but here $\delta m_{21}^{2}=5 \times 10^{-5} \mathrm{eV}^{2}$ 
$\delta m_{31}^{2}=3 \times 10^{-3} \mathrm{eV}^{2}, \delta m_{21}^{2}=10^{-4} \mathrm{eV}^{2}, \delta=\pi / 2$. We assume that each parameter including matter density has $10 \%$ ambiguity. The sensitivity is almost proportional to

$$
\left(\sin \delta \sin 2 \theta_{12} \sin 2 \theta_{23} \cos \theta_{13} \frac{\delta m_{21}^{2}}{\delta m_{31}^{2}}\right)^{2} .
$$

Therefore, the sensitivity becomes 4 times worse when $\delta m_{21}^{2}=5 \times 10^{-5} \mathrm{eV}^{2}$ (see Fig. 3).

In Fig.2, it is found that at worst $P_{\mu}= \pm 0.3$ should be needed to observe T-violation effect with a total of $10^{21}$ muon decays and a 100-kt detector. On the contrary to the sensitivity of CP-violation effect usually discussed, the sensitivity will not change drastically by including other sources of errors, since the $V$ will convey the largest error among the other uncertainties.

In the remaining of this section, we see what we can learn from the theoretical analysis.

\subsection{How many $\mu^{+}$and $\mu^{-}$decays are needed ?}

In this subsection, the most efficient ratio, $f_{i}$, between the numbers of $\mu^{+}\left(N_{\mu^{+}}\right)$and $\mu^{-}$ $\left(N_{\mu^{-}}\right)$to obtain the best sensitivity to $\mathrm{CP}$ violation is estimated for a fixed distribution of $N_{\mu^{-}}$at each polarization $P_{\mu_{i}}$. To do this, the $\chi^{2}$ can be rewritten to factor out the numbers of events as follows,

$$
\begin{aligned}
N & \rightarrow N_{\mu^{+}} N \\
\bar{N} & \rightarrow N_{\mu^{-}} \bar{N} \\
V & \rightarrow N_{\mu^{-}} V \\
\chi^{2} \rightarrow & \frac{\left(N_{0} \bar{N}-\bar{N}_{0} N\right)^{2}}{N_{0}^{2} V / N_{\mu^{-}}+\bar{N}_{0}^{2} N / N_{\mu^{+}}} .
\end{aligned}
$$

If the total numbers of muon is given, then

$$
\sum_{i} N_{i}+N_{\mu^{+}}=\sum_{i} \frac{N_{\mu^{-}}}{f_{i}}+N_{\mu^{+}}=F N_{\mu^{-}}+N_{\mu^{+}}=N
$$

is the given number of the total events. Here, $F$ is given by eq.(15). Under these conditions, the most efficient ratio of the numbers of $\mu^{+}\left(N_{\mu^{+}}\right)$and $\mu^{-}\left(N_{\mu^{-}}\right)$is given by

$$
\frac{N_{\mu^{+}}}{\sum_{i} N_{i}}=\frac{\bar{N}_{0}}{N_{0}} \sqrt{\frac{N}{A}}
$$

where $A$ is given by eq. (28) $\times F$. 


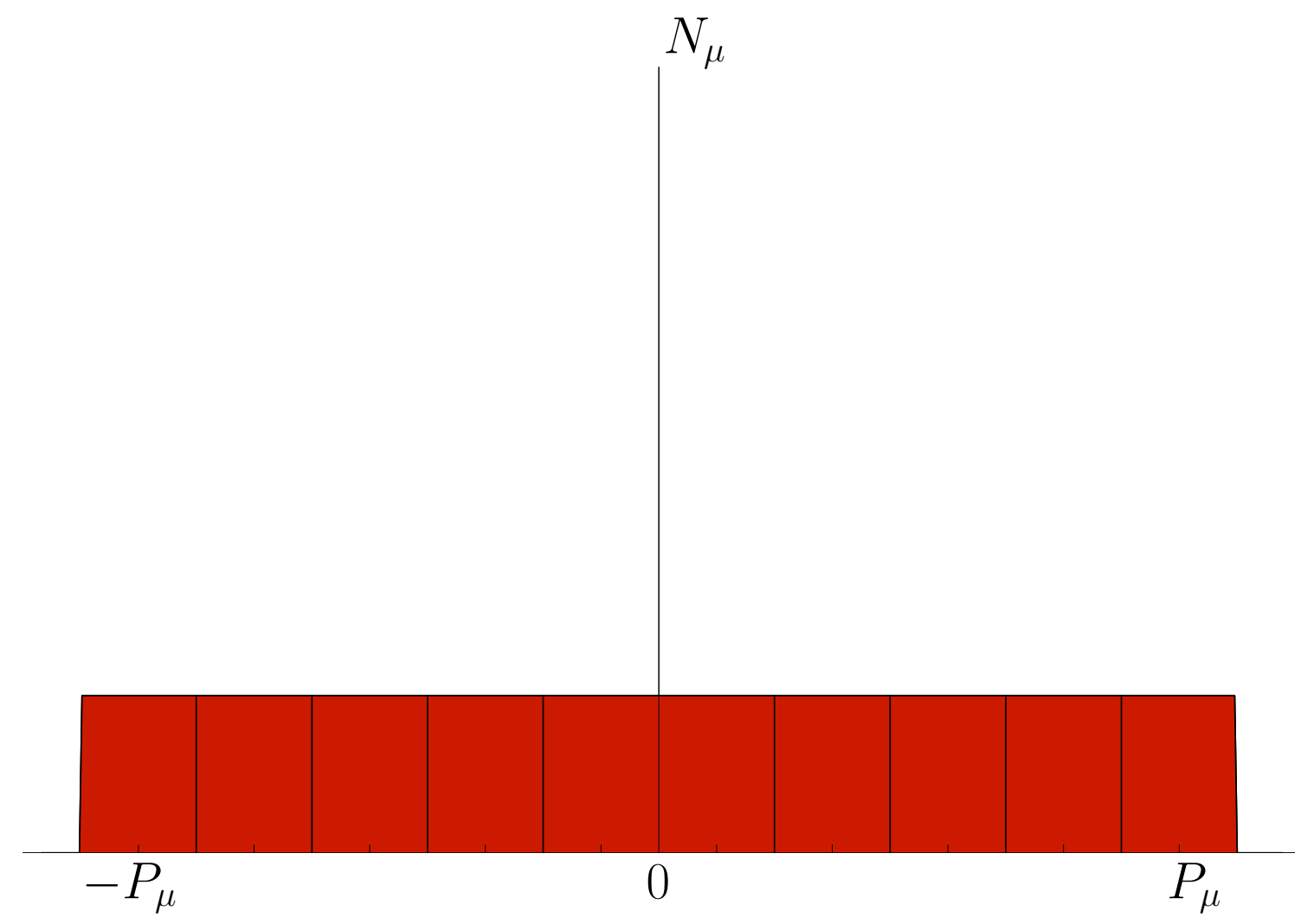

Figure 4: Uniform Distribution of polarization of muon, being distributed from $-P_{\mu}$ to $+P_{\mu}$ uniformly.

Since the oscillation probability is at most a few $\%$, while $N_{\bar{P}_{\mu}}$ in eq.(28) is essentially given by the non-oscillation event, from the fact that $N / N_{\bar{P}_{\mu}} \sim O\left(10^{-2}\right)$ and that $\sum_{i}\left[1 /\left(f_{i}^{2} y_{i}\right)\right] / \sum_{i}\left[\left(P_{\mu_{i}}^{*}\right)^{2} /\left(f_{i}^{2} y_{i}\right)\right]$ in eq.(28) is much larger than a unity in a realistic case, it is found that a much larger number of $\mu^{-}$is needed. Therefore by tuning the ratio of the numbers of $\mu^{+}$and $\mu^{-}$, a higher sensitivity to T-violation effect can be obtained by several tens $\%$.

\subsection{Dependence of Muon Polarization Distribution}

The measurements of $N_{i}$ events with muon polarization $P_{\mu_{i}}$ are usually made. In this subsection, the optimization of $N_{i} \mathrm{~s}(i=1 \cdots n)$ for each given $P_{\mu_{i}}$ to obtain the best sensitivity is studied. This is equivalent to examine the best muon polarization distribution.

For the most simple case to consider is a uniform distribution of $N_{i}$ from $-P_{\mu}$ to $+P_{\mu}$ as shown in Fig. 4 .

Let's consider two cases. One is a uniform distribution of $N_{i}$ from $-P_{\mu}$ to $+P_{\mu}$ as shown in Fig. 4 . The other is discrete measurement at $\pm P_{\mu}$ as seen in Fig.5.

It is assumed that the same numbers of muon decays are used for the two different distributions of Fig. (1 and Fig.5. In this case, the variances (28) of the extrapolated appearance numbers are different, depending on the distribution. In case of Fig.⿴囗十

\footnotetext{
${ }^{5}$ Here we assume that we do not have very large polarization. It means that $y_{i}$ 's are almost constant.
} 


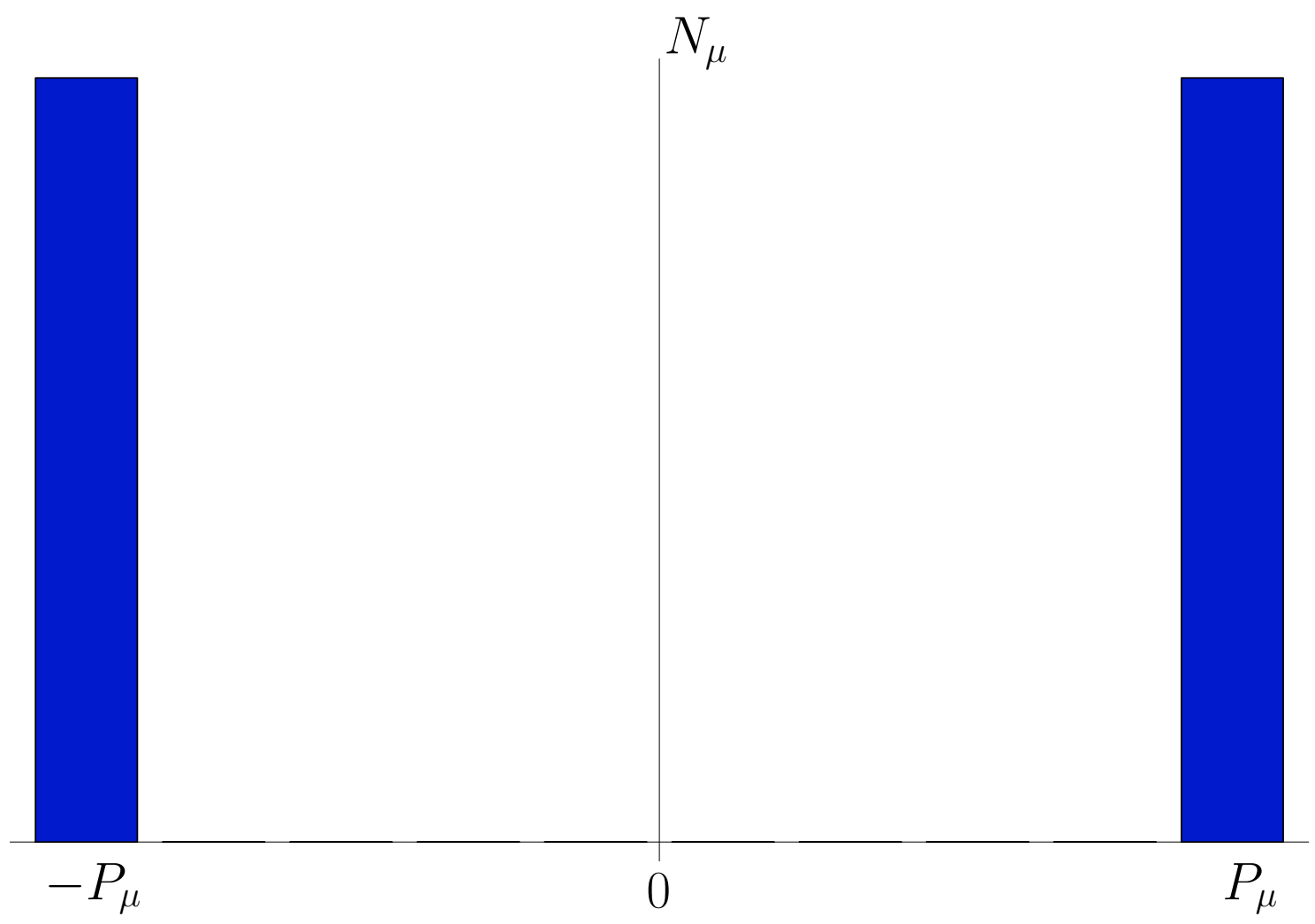

Figure 5: Distribution of the polarization of $\mu$. There are only muons with their polarization around $-P_{\mu}$ and $+P_{\mu}$. 


$$
\frac{1}{F} \frac{\sum_{i} 1 / f_{i}^{2} y_{i}}{\sum_{i}\left(P_{\mu_{i}}^{*}\right)^{2} / f_{i}^{2} y_{i}}-1 \propto \frac{3}{P_{\mu}{ }^{2}}-1
$$

while in case of Fig.5,

$$
\frac{1}{F} \frac{\sum_{i} 1 / f_{i}^{2} y_{i}}{\sum_{i}\left(P_{\mu_{i}}^{*}\right)^{2} / f_{i}^{2} y_{i}}-1 \propto \frac{1}{P_{\mu}^{2}}-1 .
$$

Therefore, a three times higher sensitivity can be obtained for the distribution of Fig.5. In reality, there will be a loss of muon decays to create the distribution in Fig.5. If this reduction factor of the number of events is defined to be $M$ (namely only $(1 / M)$ events can be used), the sensitivity is given by

$$
\frac{1}{F} \frac{\sum_{i} 1 / f_{i}^{2} y_{i}}{\sum_{i}\left(P_{\mu_{i}}^{*}\right)^{2} / f_{i}^{2} y_{i}}-1 \propto \frac{M}{P_{\mu}{ }^{2}}-1 .
$$

Then the sensitivity is better by $3 / M$. To discuss further, it is needed to have realistic muon polarization distribution. However, once it is obtained, the discussion to study a sensitivity is quite straightforward.

\section{Summary and Discussion}

In this note, we have examined a possibility to study T-violation with the use of muon polarization. The physics motivation to search for T-violation is to observe direct $\mathrm{CP}$ violation arising from the imaginary phase in the neutrino mixing matrix. The advantage of search for T-violation is to have a negligible small matter effect.

In the present study, the sensitivity to T-violation has been studied as a function of muon energy and long baseline length for various set of muon polarizations. In each set, it is assumed that at minimum three measurements with different values of muon polarization are used, for instance, $P_{\mu_{i}}= \pm P_{\mu}$ and 0 are used in the present study, where $P_{\mu}$ is the magnitude of the muon polarization. Needless to say, it is shown that the muon polarization achievable is larger, the sensitivity is better. From the present study, it is found that $\left|P_{\mu}\right|>0.3$ is necessary when it is assumed that we can get a total of $10^{21}$ muon decays and a 100-kt detector.

The magnitude of muon polarization is being studied in the accelerator study. It is not easy to have large muon polarization in a very high intensity. It would be needed that the magnitude of muon polarization and a number of muons have to be traded off. However, if search for $\mathrm{T}$ violation can be made with realistic magnitude of muon polarization, it would provide unique way to study the CP phase in the MNS leptonic mixing matrix. 


\section{Acknowledgments}

The work of J.S. is supported in part by a Grant-in-Aid for Scientific Research of the Ministry of Education, Science and Culture, \#12047221, \#12740157.

\section{References}

[1] V. Barger, K. Whisnant, and R. J. N. Phillips, Phys. Rev. Lett. 45 (1980) 2084; S. Pakvasa, in High Energy Physics 1980, AIP Conf. Proc. No.68, ed. by L. Durand, and L. G. Pondrom (AIP, New York, 1981), p.1164.

[2] J. Arafune, M. Koike, and J. Sato, Phys. Rev. D56 (1997) 3093; erratum ibid. D60 (1999) 119905; J. Arafune, and J. Sato, Phys. Rev. D55 (1997) 1653; M. Koike, and J. Sato, hep-ph/9707203.

[3] V. Barger, S. Geer, R. Raja, and K. Whisnant, Phys. Rev. D62 (2000) 013004; Phys. Lett. B485 (2000) 379; Phys. Rev. D63 (2001) 033002; Phys. Rev. D62 (2000) 073002; V. Barger, S. Geer, and K. Whisnant, Phys. Rev. D61 (2000) 053004; S. Geer, Phys. Rev. D57 (1998) 6989, erratum ibid. D59 (1999) 039903.

[4] J. B. Castell, M. B. Gavela, J. J. Gómez Candenas, P. Hernández, and O. Mena, hep-ph/0103258; A. Cervera, A. Donini, M. B. Gavela, J. J. Gómez Cadenas, P. Hernández, O. Mena, and S. Rigolin, Nucl. Phys. B579 (2000) 17; A. De Rújula, M. B. Gavela, and P. Hernández, Nucl. Phys. B547 (1999) 21; A. Donini, M. B. Gavela, P. Hernández, and S. Rigolin, Nucl. Phys. B574 (2000) 23.

[5] M. Freund, P. Huber, and M. Lindner, hep-ph/0105071; Nucl. Phys. B585 (2000) 105; M. Freund, M. Lindner, S. T. Petcov, and A. Romanino, Nucl. Phys. B578 (2000) 27; K. Dick, M. Freund, M. Lindner, and A. Romanino, Nucl. Phys. B562 (1999) 29; A. Romanino, Nucl. Phys. B574 (2000) 675.

[6] Neutrino Factory and Muon Collider Collaboration (D. Ayres et al.,), physics /9911009; C. Albright et al., hep-ex/0008064.

[7] M. Campanelli, A. Bueno, and A. Rubbia, hep-ph/9905240; A. Bueno, M. Campanelli, and A. Rubbia, Nucl. Phys. B573 (2000) 27; Nucl. Phys. B589 (2000) 577.

[8] J. Pinney, and O. Yasuda, hep-ph/0105087; O. Yasuda, hep-ph/0005134.

[9] J. Sato, hep-ph/0006127; M. Koike, and J.Sato, Phys. Rev. D61 (2000) 073012.

[10] P. Lipari, hep-ph/0102046.

[11] M. Koike, T. Ota, and J. Sato, hep-ph/0011387; J. Sato, hep-ph/0008056.

[12] T. Miura, E. Takasugi, Y. Kuno, and M. Yoshimura, Phys. Rev. D64 (2001) 013002; T. Miura, T. Shindou, E. Takasugi, and M. Yoshimura, hep-ph/0106086. 
[13] P. F. Harrison, and W. G. Scott, Phys. Lett. B476 (2000) 349.

[14] A. Rubbia, hep-ph/0106088.

[15] E. K. Akhmedov, P. Huber, M. Lindner, and T. Ohlsson, hep-ph/0105071.

[16] S. J. Parke, and T. J. Weiler, Phys. Lett. B501 (2001) 106.

[17] A. Blondel, Nucl. Instr. and Methods, A451 (2000) 131.

[18] H. Yokomakura, K. Kimura, and A. Takamura, Phys. Lett. B496 (2000) 175.

[19] A. de Gouvêa, Phys. Rev. D63 (2001) 093003.

[20] O. Yasuda, Acta Phys. Polon. B30 (1999) 3089; hep-ph/9910428.

[21] V. Barger et al., Phys. Rev. D59 (1999) 113010.

[22] S. M. Bilenky, C. Giunti, and W. Grimus, Phys. Rev. D58 (1998) 033001.

[23] S. Toshev, Phys. Lett. B226 (1989) 335; Mod. Phys. Lett. A6 (1991) 455.

[24] P. I. Krastev, and S. T. Petcov, Phys. Lett. B205 (1988) 84.

[25] T. K. Kuo, and J. Pantaleone, Phys. Lett. B198 (1987) 406.

[26] J. Sato, Nucl. Instrum. Meth. A451 (2000) 36; M. Koike, and J. Sato, Phys. Rev. D62 (2000) 073006. 